Preliminary communication UDC $130.121(045)$

1 Schmitz, H.

1 McDowell, J.

doi: $10.21464 / \mathrm{sp} 33208$

Received: November 10, 2018

\author{
Michael Meyer \\ DE-04001 Leipzig \\ michaelmeyer1749@gmx.de
}

\title{
Das Finden der unverlorenen Welt
}

\section{Bemerkungen zu einem Vergleich der Philosophien von Hermann Schmitz und John McDowell}

\begin{abstract}
Zusammenfassung
Die Philosophie von John McDowell und das neuphänomenologische Denken von Hermann Schmitz lassen sich als Therapien des Abstandes von Geist und Welt verstehen, der in der Moderne dramatische Bedeutung erlangte. In diesem Aufsatz wird versucht, die therapeutischen Ansätze von Schmitz und McDowell vor dem Hintergrund der Moderne und insbesondere als Konstrast zwischen einem phänomenologischen und analytischen Philosophieren zu vergleichen. Der Hauptunterschied in ihren Bemühungen, die Weltoffenheit des Geistes vorzustellen, ist dabei, dass Schmitz in die Beziehung zwischen Geist und Welt den Leib als zentrale Größe einbringt. Von diesem reichhaltigen Wahrnehmungsbegriff her fällt der Reduktionismus von McDowells Modell stärker auf. Dennoch wird in diesem Aufsatz auf zwei Möglichkeiten hingewiesen, Ideen von McDowell auch in einem neuphänomenologischen Kontext fruchtbar werden zu lassen.
\end{abstract}

\section{Schlüsselwörter}

Hermann Schmitz, John McDowell, Neue Phänomenologie, Weltoffenheit, Skeptizismus, Moderne

„Es gibt auch Bäume, ich kenne ihre runzlige Rinde, und Wasser, ich koste dessen Geschmack. Dieser Duft nach Gras und der Sternenschein, nachts, an bestimmten Abenden, an denen das Herz weit wird - wie könnte ich die Welt leugnen, deren Macht und Stärke ich empfinde? "1

\section{Die Welt ist da}

Philosophie zwingt sich nicht auf, sie setzt sich aus. Sie erhält sich am Leben, weil die Absurdität der Zeit, die auch sie selbst durchdringt, nach ihr verlangt. In ihr wartet das Versprechen, dass sich Intelligenz und Leben geben können, immer noch darauf, eingehalten zu werden.

Mit der Etablierung der Aufklärung im 18. Jahrhundert entstanden zuerst die tiefgreifenden Zweifel an der Lebensdienlichkeit der Vernunft, die auch im 1

Albert Camus, Der Mythos des Sisyphos, übersetzt von Vincent von Wroblewsky, Rowohlt, Reinbek bei Hamburg 2001, S. 31. 
21. Jahrhundert noch nahe gehen. Was in der Neuzeit optimistisch als selbstveredelnde Erziehung des Menschengeschlechts fortschreitet, beginnt in der Moderne weh zu tun. Die Aufklärung als Emanzipation von Traditionen durch die Aufwertung der Souveränität des Einzelnen durch das Denken, zeigt mehr und mehr ihre abgründigen Seiten. Ab 1750 werden mit Rousseaus Zivilisationskritik, mit Voltaires Reaktion auf das Erdbeben von Lissabon im Jahr 1755 - sein Candide erscheint 1759 -, mit Goethes 1774 veröffentlichtem Briefroman Die Leiden des jungen Werther, mit dem Erschrecken über den Absturz der Franzöischen Revolution in den ,terreur" ab 1793 und mit der Sehnsucht der Romantik nach einem neuen Mythos die Bedenken an der prästabilen Harmonie von Geist und Welt expliziter.

Die Geschichte als Gewalt und die Individualität als Leere: Das sind die massgeblichen Erfahrungen, die in der Moderne letztlich zu einem Zweifel des Geistes an sich selbst führen. Die emanzipierte Subjektivität entdeckt sich selbst als das eigentliche Problem. Die Aufklärung der Aufklärung entsteht unter dem Verdacht, dass die Wirklichkeit nicht nur etwas Anderes ist als bestimmte Konzeptionen, die wir uns von ihr machen, sondern dass schon das Machen von Vorstellungen die Realität verstellt. So wirken die Interpretationen des Geistes, die Welt verändern sollen, mehr und mehr wie autohypnotische Parolen der Verklärung von Gewalt. Und der Geist selbst wird sich auffällig als Automatismus eines zwanghaften Gedankenmachens. Eine eindrückliche Stelle aus Sartres Der Ekel lässt diese Erfahrung zu Wort kommen:

„[W]enn ich bloß aufhören könnte zu denken, das wäre schon besser. Die Gedanken sind das Fadeste, was es gibt. (...) Das zieht sich endlos in die Länge (...) und das hört nie auf. (...) Wenn ich mich daran hindern könnte zu denken! (...) Ich will nicht denken... Ich denke, daß ich nicht denken will. (...) Wird das denn nie ein Ende nehmen? (...) Ich bin es, ich bin es, der mich aus dem Nichts zieht, nach dem ich trachte (...). Es schlägt halb fünf. Ich stehe auf, mein kaltes Hemd klebt an meinem Fleisch. Ich gehe hinaus. Warum? Nun, weil ich keinen Grund habe, es nicht zu tun. Auch wenn ich bleibe, auch wenn ich mich still in die Ecke kauere, werde ich mich nicht vergessen. Ich werde dasein, ich werde auf dem Fußboden lasten. Ich bin.“2

Der bei Descartes noch metaphysisch eingehegte Skeptizismus mit seinem methodischen Zweifel an allem, erfährt mit dem Voranschreiten der Moderne eine dramatische Entfesselung. Von Fremde umgeben und haltlos in sich selbst, erhält für den Geist die Idee, dass die Welt vielleicht nur in unserem Kopf existiert, eine schicksalhafte Bedeutung; bekannt ist Kleists „Kant-Kri-

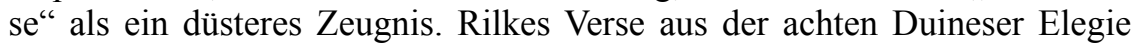
drücken den epistemischen Krisenzustand der Moderne paradigmatisch aus:

„Was draußen ist, wir wissens aus des Tiers

Antlitz allein; (...)

Dieses heißt Schicksal: gegenüber sein

und nichts als das und immer gegenüber.

(...)

Zuschauer, immer, überall,

dem allen zugewandt und nie hinaus! ${ }^{3}$

Aus einem Abstand heraus könnte die Moderne vielleicht einmal aber auch als Epoche erscheinen, bei der die Dominanz des Wirklichkeitsbegriffs des Schweren, Harten, Ernsten ein Gegengewicht bekam in den Wahrheiten des Leichten, Entlasteten, Erfinderischen. Als ein solches ,expandierendes transkulturelles Levitationsexperiment" entdeckte sich Zivilisation neu als ein „Vielkammersystem aus Auftriebszellen“. 4 Mit dieser Weitwinkelperspektive ist es möglich, die Moderne nicht nur als Mischung von Totalitarismus und 
Depression sichtbar werden zu lassen. Die Philosophie des 20. Jahrhunderts lieferte für diese Sicht mitunter entscheidene Gedanken. Wesentlich dafür ist die Revision eines dualistischen Bildes von Geist und Welt. Die verbindene These der drei Hauptströmungen der „kontinentalen“ Philosophie - der Phänomenologie, der Hermeneutik und der Ontologie - lautet dabei: Es gibt keinen Abstand des Geistes zur Wirklichkeit. Die verloren geglaubte Welt war nie verloren. Die Welt ist da.

Die von Gadamer formulierte Fassung dieses Gedankens in der Hermeneutik besagt: Wir stecken immer schon in einer Geschichte eines gegebenen Sinns, die uns hat und in die wir deutend-verstehend einrücken. Die phänomenologische Revision von Husserl lautet: Weil das Bewusstsein als intentionales strukturell immer schon Bewusstsein von etwas ist, ist der Geist konstitutiv zur Welt. Die ontologische Revision von Heidegger lehrt schließlich das „Inder-Welt-Sein“ als Merkmal des „Daseins“.

Dabei kommt es in Heideggers Sein und Zeit zu einer Übernahme des von Scheler in anthropologischem Zusammenhang gebrauchten Ausdrucks „Weltoffenheit“" als zentralen Begriffs für die nichtdistanzierte Verfassung des Geistes zur Welt. Heidegger deutet „Weltoffenheit“ derart, dass das Dasein von Menschen durch mediales Gestimmtsein vorzustellen sei:

„Die Gestimmtheit der Befindlichkeit konstituiert existenzial die Weltoffenheit des Daseins.“5

Allerdings hilft diese Idee einer intimen Weltoffenheit wie sie Heidegger denkt und wie sie der Existenzialismus fortsetzte, dem modernen Subjekt nicht viel weiter. Auch wenn das Selbstverständnis einer denkenden Zuschauerindividualität widerlegt ist: Der Geist als Teilnehmer der Welt, erfährt seine Beziehung zu ihr als Angst, Langeweile, „Seinsverlassenheit“ (Heidegger), als Ekel (Sartre), als Absurdität (Camus).

Die Neue Phänomenologie von Hermann Schmitz nun arbeitet auch einem Begriff der Weltoffenheit des Geistes zu. Indem sie den „Leib“ in das Verhältnis von Geist und Welt einbringt, weitet sich das Weltsein des Geistes zu einer „leiblichen Kommunikation": Welt ist das, was einem in seinem geistigen Besinnen auf sein Sichfinden in seiner Umgebung auf leibliche Weise unwillkürlich nahegeht. Weltoffenheit erscheint so als Vielfalt von sich lebendig durchdringenden und vermischenden somatischen Spannungen und Atmo-

2

Jean-Paul Sartre, Der Ekel, Rowohlt, Reinbek bei Hamburg 1994, S. $115 \mathrm{f}$

3

Rainer Maria Rilke, Duineser Elegien, Insel Verlag, Frankfurt a. M. 2000, S. 65.

Peter Sloterdijk, Schäume, Suhrkamp, Frankfurt a. M. 2004, S. 722f. Sloterdijk bemüht sich in seinem „Sphären“-Projekt altenative Beschreibungen für die Moderne zu finden, die die auf das Katastrophische und Leidende fixierte Deutungen relativieren. Dabei kommt es zu einer nichtverächtlichen Interpretation der westlichen „Wohlstandsblasen“ als Pluralismus von kultur- und zivilisationsanimierenden Interaktionsmöglichkeiten, mitsamt einer Auflösung von vormodernen Lebensgefühlen. Vgl. Peter Sloterdijk, Was geschah im 20. Jahrhundert?, Suhrkamp, Frankfurt a. M.
2016, S. 122: „Die Realitätsgefühle der Menschen im agro-imperialen Weltalter waren auf Güter und Ressourcenknappheit geeicht, weil ihnen die Erfahrung zugrunde lag, die Arbeit, verkörpert im mühseligen Ackerbau, reiche gerade so aus, um prekäre Inseln der humanen Künstlichkeit in die Natur zu stellen. (...) Diese Anschauungen gelten nicht mehr, seit mit dem Durchbruch zum fossilenergetischen Kulturstil vor etwas mehr als 200 Jahren ein unheimlicher Liberalismus auf die Bühne trat. (...) $[\mathrm{M}]$ an darf Verschwendung inzwischen ruhigen Tones als erste Bürgerpflicht bezeichnen. (...) Die moderne Zivilisation beruht (...) auf dem konstanten Zufließen einer unverdienten Fülle von Energie in den Unternehmens- und Erlebnisraum.“

5

Martin Heidegger, Sein und Zeit, Max Niemeyer, Tübingen 1953, S. 137. 
sphären, ausgehend von einer ,,primitiven Gegenwart“ in den unentfalteten Dimensionen Sein, Ich, Jetzt, Hier, Dieses.

Der ingeniöse Entwurf von Schmitz kann dabei als eine entscheidene Kurskorrektur moderner Selbst- und Welterfahrung interpretiert werden. Mit ihm ist es nicht nur möglich, Heideggers monomanische Ausrichtung auf bestimmte "Grundstimmungen“ zu widerlegen, die hermeneutische Sinngeschichte zu intimisieren und Husserls Intentionalität als zu einseitige Bindung aufzulösen. Die Neue Phänomenologie widerlegt zudem therapeutisch den in Rilkes Zeilen verkörperten Grundzustand moderner Subjektivität als eines lebenslangen Gegenüberseins. Der Dauerzustand des Draußenseins kann mit Schmitz als Folge der Entfremdung einer leiblich verarmten Kultur angesehen werden, in der eine eingespielte ,entfaltete Gegenwart“ von Geist, Leib und Welt noch aussteht.

Mit dem Ansatz bei einem leiblich-besinnlichen Geist richtet sich Schmitz auch gegen weite Bereiche des zeitgenössischen Denkbetriebs und vor allem gegen die analytische Philosophie. Es fehlt ihm darin die für ihn zentrale leibliche Subjektivität. An die Stelle eines affektiven Betroffenseins der ersten Person rückt in der analytischen Philosophie, die für die Dramatiken der Modernen unempfänglich ist, ein neutralisiertes Beobachten einer „,view from nowhere" (Thomas Nagel). Damit steht das Denken der analytischen Philosophie für Schmitz unter dem Verdacht ,eine Philosophie gegen die Philosophie" zu sein:

„Die analytische Philosophie hat durch kritische und analytische Genauigkeit in Einzelfragen und durch Einübung kontrollierter Diskussion unter Forschern wichtige Verdienste, aber als Erbe des philosophischen Positivismus in unserer Zeit hat sie von Mach, Avenarius, der Wiener Schule und Wittgenstein die Verdrängung der Subjektivität übernommen (...). Die Philosophie, verstanden als ein Sichbesinnen des Menschen auf sein Sichfinden in seiner Umgebung, ist aber nur sinnvoll im Ausgang von der Subjektivität, und daher ist die analytische Philosophie sozusagen eine Philosophie gegen die Philosophie."66

Dieser Verdacht der Neuen Phänomenologie gegen die analytische Philosophie läßt sich nun in einem Zusammenhang bringen mit dem Denken John McDowells. Interessant ist dieser Vergleich, weil McDowells Denken vom Boden der analytischen Philosophie her eine grundsätzliche Öffnung der reinen Analytik zur wahrnehmenden Subjektivität hin bedeutet. Eine gewisse Berühmheit erlangte dabei seine Formulierung, dass ein Verständnis des Denkens ohne das Wahrnehmen miteinzubeziehen zu einem fatalen Bild des Denkens führt, dass als ein „frictionless spinning in a void“"7 vorgestellt werden muss. Darüber hinaus verfolgt McDowell einen therapeutischen Ansatz, der sich der modernen Problematik der Kluft von Welt und Geist bewusst ist. Sein Versuch einer Schlichtung der Dualität, so wird zu zeigen sein, lässt sich zwar mit der Neuen Phänomenologie verbinden, weicht aber auch gravierend von ihr ab.

Im Folgenden möchte ich zunächst McDowells Versuch einer Öffnung zur wahrnehmenden Subjektivität vorstellen, die er im Hinblick auf ein nichtskeptisches Verhältnis von Geist und Welt entwirft. Im Anschluss daran soll der Unterschied zur Neuen Phänomenologie aufgezeigt werden und der Frage nachgegangen werden, inwiefern sich mit McDowells Denken womöglich eine Gelegenheit bietet, die Abstände von Neuer Phänomenologie und analytischer Philosophie zu überbrücken - oder zu erweitern. ${ }^{8}$ Zuletzt möchte ich den Versuch wagen, die Neue Phänomenologie zu würdigen, indem ich sie skizzenhaft einordne in die gegenwärtige Lage der Philosophie. 


\section{McDowells therapeutischer Realismus}

\section{1. "to exorcize not answer"}

Um das Denken von John McDowell vorzustellen, werde ich sein epochemachendes Werk Mind and World kurz skizzieren, insofern es das Thema von Denken und Wahrnehmen im engeren Sinne betrifft. McDowell gelingt es in diesem Buch, das analytische Denken für den Horizont der wahrnehmbaren Welt zu öffnen, indem er die grundsätzliche Kooperation von Denken und Wahrnehmen vorstellbar macht. Allerdings tut er dies, ohne diesen Horizont zu betreten oder auszuleuchten.

McDowell geht es um die Auflösung des skeptizistischen Gegensatzes von Geist und Welt und den aus ihm stammenden Folgedualismen - Geist und Natur, Natur und Kultur, Begriff und Anschauung, Erkennen und Normativität, Rechtfertigen und Wahrnehmen. McDowells Zurückhaltung in Bezug auf einen differenzierten Begriff von Wahrnehmung ist daher weniger ein Mangel, als eine Folge seines philosophischen Programms. Ihm geht es darum, Gedanken zu offerieren, mit denen Interpretationen skeptizistischer Gegensätze gegeben werden, die diese unnötig erscheinen lassen.

McDowells therapeutisches Philosophieren bezieht sich so weniger auf den epistemischen Fragenbereich, der sich bemüht, den Bezug des Geistes zur Welt zu denken. Zentral ist für ihn vielmehr die Frage, nach der Bedeutung der grundsätzlichen Frage, ob im Denken überhaupt eine Beziehung zur Wirklichkeit gegeben ist. In dieser Fragestellung sieht er eine „,deeper anxiety“ der modernen Philosophie:

„It is true that modern philosophy is pervaded by apparent problems about knowledge in particular. But I think it is helpful to see those apparent problems as more or less inept expressions of a deeper anxiety - an inchoately felt threat that a way of thinking we find ourselves falling into leaves mind simply out of touch with the rest of reality, not just questionably capable of getting to know about it."

Indem McDowell der Frage in therapeutischer Manier nachgeht, ob das Denken ,out of touch with the rest of reality“ ist, gewinnt seine Philosophie Kontur als ein antiskeptizistischer Realismus. Dafür widmet er sich der skeptizis-

Hermann Schmitz, Höhlengänge. Über die gegenwärtige Aufgabe der Philosophie, Akademie Verlag, Berlin 1997, S. 18.

John Henry McDowell, Mind and World, Harvard University Press, Cambridge (MA) 1996, S. 11. Im Folgenden wird auf diese erweiterte Ausgabe von Mind and World, ursprünglich 1994 erschienen, Bezug genommen, die direkt im Fließtext mit der Sigle „MW“ zitiert wird.

8

Für eine umfassendere Version der hier vorgetragenen Gedanken, die sich dort aber näher an McDowells Denken orientieren, vergleiche vom Verfasser: Michael Meyer, Die Welt denken. Versuch einer kritischen Reformulierung von McDowells Begriff der Offenheit des Geistes zur Welt (Dissertation), Berlin Freie Universität, Berlin 2017.
MW, S. xiii.

10

McDowells Ansatz kann auch als Verteidigung des vortheoretischen common sense gegenüber der Philosophie verstanden werden, worin er der Neuen Phänomenologie ähnelt: „Of course if that is our predicament, we need to answer the traditional sceptical questions before we can talk of openness to the world. But my talk of openness to the world is a rejection of the traditional predicament, not an attempt to respond to it. (...) The aim is here not to answer sceptical questions, but to begin to see how it might be intellectually respectable to ignore them, to treat them as unreal, in the way that common sense has always wanted to." Ebd., S. 112f. 
tischen Frage, indem er nicht direkt auf sie eingeht. Der Grund dafür ist, dass McDowell in der epistemischen Frage nach der Erreichbarkeit der Welt durch den Geist eine metaphysische Überzeugung wittert, die einen Abstand zwischen Geist und Welt voraussetzt. So ist für McDowell das Problematisieren des Skeptizismus das eigentliche Problem. Der Skeptizismus gräbt implizit an dem Graben, den er explizit überbrücken möchte. Jegliches Denken, das den Themen des Skeptizismus folgt, bejaht daher unbedacht den Rahmen, der dieses Fragen sinnvoll erscheinen lässt. ${ }^{10}$

Die von der skeptischen Fragehaltung ausgehenden Problematiken sind für McDowell so allesamt Ausdruck einer reflexiven Verstiegenheit innerhalb eines verfehlten „frame of mind“. Sie sind unnötig kompliziert. Ihre Verstiegenheit will er aufzeigen und auflösen:

[Es geht ihm darum] ,... to exorcize (not answer) the questions that give expression to that distinctively philosophical kind of puzzlement, the kind that issues from a frame of mind that, when fully explicit, would purport to display an impossibility in what the questions are asked about."11

Durch diesen therapeutischen Ansatz verfestigt McDowell nicht die Plausibilitäten, aus denen ein skeptisches Fragen entspringt. Er dekonstruiert den Rahmen, in dem eine Kluft von Welt und Geist als sinnvoll anzusehen ist. Sein Ziel ist es, dass, wenn es gelingt, einen wenn auch nur minimalen Spalt einer ,world-directedness as such“12 plausibel zu machen, sämtliche Theorien und ihre Diskussionen an Bedeutung verlieren, die auf den skeptizistischen Prämissen beruhen. McDowells Philosophie möchte also ein überzeugendes Modell dafür liefern, dass der Geist unmittelbar zur Welt ist und damit die Modelle eines Abstandes von Welt und Geist widerlegen.

Der für einen möglichen Dialog mit der Neuen Phänomenologie wichtige Aspekt der von McDowell thematisierten Verbindung von Wahrnehmung und Denken ist jedoch nicht überzubewerten. McDowell verfolgt lediglich eine therapeutische, antiskeptische Philosophie, die nicht im Detail an der Darstellung des epistemischen Wertes der Wahrnehmung oder auch der Leiblichkeit orientiert ist. Er schlägt an neuralgischen Stellen schlichtende Umdeutungen vor, die aber wiederum auch nur die für die analytische Philosophie massgeblichen Theoriestränge und Debatten betreffen.

\subsection{Das Urteil der Welt}

McDowell entwickelt seine Gedanken in den Themenstellungen und Vokabularen des analytischen Diskurses. Die neuzeitliche Distanz von Geist und Welt stellt sich dabei in dem Gegensatz von Sinneseindrücken und begrifflichem Gehalt heraus. So versucht McDowell einerseits einen von ihm so genannten „Mythos des Gegebene“ zu vermeiden, der den Sinneseindrücken einen nichtbegrifflichen Gehalt zuschreibt und bloße Anschauungen als Gründe für Urteile ansieht. Andererseits grenzt er sich aber auch ab gegen einen „Kohärentismus", der lediglich ein Interagieren als ein Folgern von Urteilen in Begründungsketten für ein Erlangen von Wahrheit für nötig hält. Der Einfluß der erfahrbaren Welt wird dabei entwertet zu einer bloßen Kausalität.

Für die Formulierung seines „minimalen Empirismus“ verbindet McDowell beide Positionen, indem er kritisch ihre jeweilige implizite Kluft von Geist und Welt herausstellt. So berücksichtigt der „Mythos des Gegebenen“ seiner Meinung nach zwar die subjektive Wahrnehmung, hat aber das Problem, dass eine Orientierung am Gegebenen nicht die Kluft überbrücken kann, die zwischen einer arationalen Erfahrung und einer begründeten Meinung liegt. 
Wenn man Perzeptionen einen Gehalt zuschreibt, der nicht-propositional ist, dann ist es, laut McDowell, nicht klar, wie dieser Gehalt je geistige Gehalte beeinflussen kann. Es ist undeutlich, wie perzeptiver Gehalt je zu einem Glied in einer Begründungskette wird. Die Reibung zur Welt kann im „Mythos des Gegebenen" nicht in den Geist übersetzt werden.

Anders stellt sich das Problem der Verbindung von Geist und Welt im „Kohärentismus" dar. Allein an Kohärenz orientiert, kann ein solcher Ansatz nicht klären, inwiefern die aufeinander inferentiell bezogenen konzeptionellen Gehalte als welthaft zu begreifen sind. Es ergibt sich ein Formalismus, der die Reibung zur Welt nicht vorstellen kann.

McDowells „minimaler Empirismus“ versucht nun eine Kooperation von Rezeptivität und Spontanität als Kombination des „Mythos des Gegebenen“ und des „Kohärentismus“ zu formulieren. Dabei muss die sinnliche Wahrnehmung als ein Empfangen von ungeformten, rohen Sinnesdaten, die dann nachträglich begrifflich schematisiert werden, anders verstanden werden. ${ }^{13}$ Dazu gehört es auch, als ob das begriffliche Denken nicht so gedacht wird, dass es als eine repräsentationale Vermittlungsstufe zwischen Geist und Welt träte.

McDowells erster Schritt bei seiner auflösenden Umdeutung besteht darin, gegen den Skeptizismus anzunehmen, dass das Wahrnehmen grundsätzlich die Welt erfassen kann. McDowell begründet diesen Gedanken mit Hilfe einer therapeutischen Idee: Ohne die Annahme der Welthaltigkeit des Wahrnehmens macht auch der Begriff des Denkens keinen Sinn. Wenn das Wahrnehmen nicht als ein unmittelbares Erkennen von Welt begriffen wird, dann kann das Denken nicht als etwas verstanden werden, was die Realität erfasst. Es muss dann als ein „frictionless spinning in a void“ ${ }^{\prime 14}$ vorgestellt werden.

McDowell reagiert mit dieser Abgrenzung zum „Kohärentismus“ auf die skeptische Sorge darüber, dass auch wenn alle Überzeugungen geistig intern kohärent sind, es noch längst nicht ausgemacht ist, ob der Geist damit die externe Wirklichkeit berührt. Spontanität muss so verstanden werden, dass sie von Rezeptivität kontrolliert werden kann. Oder anders gesagt: Das Denken muss offen sein für das Wahrnehmen. Rationalität ist so aufzufassen, dass sie nicht nur von Gründen, sondern, und wenn auch nur in einem minimalen Sinn, von Wahrnehmungen bestimmt wird:

„That is what I mean by , a minimal empiricism": the idea that experience must constitute a tribunal, mediating the way our thinking is answerable to how things are, as it must be if we are to make sense of it as thinking at all."15

Die Tribunalisierungsfunktion der Wahrnehmung sieht McDowell als die Kraft des Einspruchs der Welt gegen das Denken. Die Annahme der Weltun-

11

Ebd., S. xxi.

12

Ebd., S. xiv.

13

McDowell stimmt dabei Davidson zu, der den Dualismus von Schema und Material als drittes Dogma des Empirismus kritisiert. Demnach wird das Erfahren nicht so begriffen, dass ein „Begriffsschema“ auf sinnlichen Input angewendet wird. Vgl. Donald Davidson, "On the Very Idea of a Conceptual Scheme“, Inquiries into Truth and Interpretation, Cla- rendon Press, Oxford 2007, S. 183-198 Davidsons theoretisches Setting ist aber ein anderes als McDowells, so dass beide Argumentationen nur grob verglichen werden können. Verkürzt gesagt: Davidson geht es um die Interpretation von Kommunikation als Kohärenz, McDowell um die Rezeptivität als eine Dimension von Kohärenz.

14 MW, S. 11. 15 Ebd., S. xii. 
mittelbarkeit der Wahrnehmung wird dabei präzisiert. Die wahrgenommene Welt muss nicht nur als etwas begriffen werden, was in der Wahrnehmung gegeben ist. Sie muss auch so gegeben sein, dass sie als autonom vom Geist verstanden werden kann. Wahrnehmen muss daher passiv sein. McDowell denkt diese Passivität als Einschränkung der Aktivität des Denkens. ${ }^{16}$ Es liegt außerhalb der Macht des Denkens, was erfahren wird. Die Welt lässt dem Denken keine Wahl:

„In experience one finds oneself saddled with content. One's conceptual capacities have already been brought into play, in the content's being available to one, before one has any choice in the matter. The content is not something one has put together oneself, as when one decides what to say about something. (...) [E]xperience is passive (...)." ${ }^{\text {17 }}$

In diesen Formulierungen findet sich auch schon McDowells weitere Entfaltung des therapeutischen Realismus. Das Wahrnehmen als regulierende Kraft über das Denken darf nicht so begriffen werden, als stünde es dem Denken gegenüber. Wenn das Wahrnehmen als etwas begriffen werden soll, was das Denken auch in der Form einer Überzeugung beeinflussen kann, so muss eine Erklärung dafür gefunden werden, wie Wahrnehmungen als eine Macht über das Inferentielle auch als inferentiell verstanden werden können. Streng genommen können Wahrnehmungen im analytischen Diskurs nämlich nur Behauptungen beeinflussen, wenn sie einen konzeptionellen, genauer: propositionalen Gehalt besitzen. Nötig ist so die Annahme eines empirischen propositionalen Gehaltes, wenn das Wahrnehmen die Kraft eines Tribunals gewinnen soll. Damit steht McDowell vor dem Problem, Wahrnehmungen als Behauptungen zu verstehen.

McDowell löst dieses Problem therapeutisch auf, indem zum einen Wahrnehmungen als formal normativ interpretiert werden. Sie können mit Überzeugungen interagieren, weil sie so gedeutet werden können, dass sie der Form nach Urteile sind. Zudem deutet er Spontanität als etwas, das nicht bloß als aktives Urteilen begriffen werden muss. Nach McDowell ist die Anwesenheit der begrifflichen Fähigkeit in der Wahrnehmung derart zu denken, dass sie in der Wahrnehmung zwar präsent, aber passiv präsent ist. Da die Fähigkeiten der Konzeptionalität von ihm so interpretiert werden, dass sie immer schon im Wahrnehmen passiv wirken, stellt das Denken diese nur explizit heraus. Wahrnehmen und Denken sind so der Form nach identisch, unterscheiden sich aber im Modus von einander.

McDowell kann durch die Idee der passiven Anwesenheit des Denkens in der Wahrnehmung erklären, dass das Wahrnehmen zwar urteilskompatibel ist, aber kein permanentes Urteilen zu sein braucht. Als solches wäre es eine zweite Ebene des Erfahrens, die auf ein dualistisches Verständnis schließen ließe, bei dem das Denken eine Art Kommentar zu dem Wahrnehmen darstellte.

Mit dem Gedanken der Anwesenheit der konzeptionellen Fähigkeiten in der Wahrnehmung weicht McDowell dem „Mythos des Gegebenen“ aus. Indem er nicht einen epistemischen Sprung aus dem Wahrnehmen in das Denken annimmt, so als wandle sich ein Erfahrungsgehalt in einen geistigen Gehalt, sondern das Wahrnehmen insgesamt für eine begriffliche Größe, nämlich als Form, erklärt, löst er den Dualismus von sinnlicher Wahrnehmung und begrifflichem Erkennen auf. ${ }^{18}$ McDowell bindet Welt und Geist so über ein Neuverständnis von Rezeptivität aneinander. Die Welt wird konzeptionell erfahren. Wahrnehmung ist begriffsförmig:

„The relevant conceptual capacities are drawn on in receptivity. (...) It is not that they are exercised on an extra-conceptual deliverance of receptivity." ${ }^{19}$ 
So verstanden sind Wahrnehmungen für McDowell nicht Vermittler von Welt. Durch Wahrnehmungen hindurch vermag sich die reale Welt als artikulierbares Urteil zu zeigen. Das bedeutet für ihn Weltoffenheit als „fallible openness"“.20 Die Welt ist offen dafür, in Gedanken einzugehen und das Denken bleibt offen für den Einfluss der Welt.

McDowell reicht es, bis zu diesem Punkt zu kommen. Wie genau das Verhältnis von Denken und Wahrnehmen vorzustellen ist, dieser Aufgabe stellt er sich nicht. Ihm genügt es zu zeigen, dass das dualistische Setting des Skeptizismus nicht kohärent ist. Denken kann sagen, was der Fall ist:

„But to say there is no gap between thought, as such, and the world is just to dress up a truism in high-flown language. All the point comes to is that one can think, for instance, that spring has begun, and that very same thing, that spring has begun, can be the case. That is truistic, and cannot embody something metaphysically contentious, like slighting the independence of reality. “21

\subsection{Feinkörnige Begriffe}

Für eine Auseinandersetzung zwischen dem Denken McDowells und der Neuen Phänomenologie ist die Reaktion von McDowell auf einen Einwand wichtig, der gegen seine Deutung der Begriffsförmigkeit der Wahrnehmung erhoben wird. Das sogenannte „Feinkörnigkeitsargument“" spielt auf die phänomenale Erfahrung an, dass Wahrnehmungen viel feinkörniger erscheinen als die darauf referierenden Begriffe. ${ }^{22}$ Die sinnlichen Differenzierungen sind demnach mit begrifflichen Differenzierungen nicht einzuholen. Daraus wird gegen McDowell die These abgeleitet, dass der Gehalt von Wahrnehmungen nicht absolut begrifflich sein kann, weil Wahrnehmungen zu nuanciert sind. Durch diese Kritik wird McDowell dazu gedrängt, seinen therapeutischen Ansatz zu überschreiten. Es kommt zu einer konstruktiven Explikation seiner Beschreibung von Geist und Welt, an die dann ein neuphänomenologisches Denken ansetzen kann.

Gegen den Vorwurf der fehlenden Feinkörnigkeit des begrifflichen Erkennens wehrt sich McDowell mit dem Verweis auf den demonstrativen Gebrauch von Begriffen. Dieser genügt aus seiner Sicht, um die Feinheit der erfahrbaren Welt als eine konzeptionelle Feinheit zu verstehen. In der Gegenwart von sinnlichen Unterschieden lassen sich für McDowell beliebig feine Begriffe als Demonstrativa bilden, die diese Unterschiede anzeigen:

16

McDowell betont vor allem die negierende Bedeutung der erfahrenden Welt, wenn er die nötige Gebundenheit des Denkens an das Wahrnehmen beschreibt. $\mathrm{Zu}$ ergänzen wäre hierbei, dass die Wahrnehmung auch als Bekräftigung und als Auslöser von Urteilen gelten muss.

17

MW, S. 10.

18

Hierzu passt auch, dass McDowell Putnams berüchtigten Ausspruch - ,,[M] eanings“ just ain't in the head." - aufgreift: ,[T]he mind (...) is not in the head either." Siehe: John Henry McDowell, „Putnam on Mind and Meaning“, Meaning, Knowledge, and Rea- lity, Harvard University Press, Cambridge (MA) 1998, S. 275-291; hier S. 276.

19

MW, S. 9

20

Ebd., S. 143.

21

Ebd., S. 27.

22

Das Argument der Feinkörnigkeit leitet sich im Kontext der analytischen Diskurse her aus Gareth Evans, The Varieties of Reference, Oxford University Press, Oxford 1982, S. 229. 
„In the throes of an experience of the kind that putatively transcends one's conceptual power - an experience that ex hypothesis affords a suitable sample - one can give linguistic expression to a concept that is exactly as fine-grained as the experience, by uttering a phrase like, that shade", in which the demonstrative exploits the presence of the sample. “23

Auch wenn die Töne einer Geige, die Lichtspiegelungen eines Flusses, die Formen von Schneeflocken anscheinend viel zu nuanciert sind, um durch Worte gesagt werden zu können: Nach McDowell kann durch einen demonstrativen Begriff, - „Dieser Ton!“”, „Diese Lichtspiegelung!“, „Diese Schneeflocke!" - ein Differenzierungsgrad der Sprache erreicht werden, der mit den Nuancen der Welt mithalten kann.

McDowell betont so die Situationsabhängigkeit der Begriffe. Die Idee dahinter ist wieder therapeutisch motiviert. Er wendet sich gegen das dualistische Bild einer Kluft zwischen Gedanken und Welt. Diese Kluft wird nicht mehr zu einer zwingend notwendigen Annahme, wenn es Gedanken gibt, die von der Welt abhängen. Diese Abhängigkeit begreift McDowell nicht im Sinne eines Wahrheitswertes. Nicht, ob eine Aussage zutrifft oder nicht, ist entscheidend, sondern dass ein Gedanke überhaupt nicht sein könnte, wenn die Welt nicht die Beschaffenheit hat, von der er handelt. Die Welt ist so eine Existenzbedingung des Gedankens, die Bedingung der Möglichkeit seines Gedachtwerdenkönnens. McDowell nennt derartige Gedanken ,singular thoughts“ ${ }^{24}$ Ein singulärer Gedanke meint, ,a thought that would not be available to be thought or expressed if the relevant object, or objects, did not exist" .25

Die typische Ausdrucksform eines ,singular thought“ muss McDowell nun aber gemäß seiner therapeutischen Vorgaben bestimmen. Genau an dieser Stelle wird sein Verständnis von Wahrnehmung deutlich. McDowell interpretiert ein situationsabhängiges, feinkörniges Benutzen der Begriffe nämlich wie ein Zeigen auf Dinge, die in der Umgebung des Sprechenden existieren. Das Gegebene ist für ihn in der Erfahrung immer als ,,a particular bit of the Given“" ${ }^{26}$ gegeben. Dadurch fordert dieses Konzept von Realismus auch eine Welt, die allein gegenständlich verstanden werden kann. Die Welt wird alles, was zeigbar ist. McDowells Idee der Begriffsförmigkeit der Wahrnehmung ist damit genauer als Idee der Zeigbarkeit des Wahrnehmbaren zu lesen:

,[I]t must be possible to attribute to pointing, if we are to be able to assure ourselves that our conception of thinking sufficiently acknowledges the independence of reality. It must be possible for justifications of judgements to include pointing out at features of the world, from what would otherwise risk looking like a closed circle within our exercises of spontaneity run without friction." ${ }^{27}$

\section{Auf neuphänomenologischer Fahrt im dämonischen Gebiet}

\subsection{Nahegehen}

Wie läßt sich nun genauer eine mögliche Verbindung von McDowells zu Schmitz' Denken ziehen? In einer moderaten Lesart könnte man Schmitz' Ansatz als breite Ausführung der von McDowell angedachten Kooperation von Denken und Wahrnehmen begreifen. In seinem Werk würde dann McDowells Ansatz, die sinnliche Wahrnehmung epistemisch aufzuwerten, explizit verlängert zu einer Darstellung der vielfachen Verwobenheit von Geist und Welt. Die bei McDowell unscharf gebliebenen Gedanken hinsichtlich einer ,bodily presence in the world“ ${ }^{\prime 28}$ manifestierten sich bei Schmitz in überragender Differenziertheit.

Gegen diese Lesart spricht die von Schmitz explizierte Vielfalt der wahrnehmbaren Welt, mit ihren „bedeutsamen Situationen“ und den Gefühlen als 
raumhaften „Atmosphären“. Dagegen spricht auch das subjektivere Verständnis von Wahrnehmung als ,eigenleibliches Spüren“ der „leiblichen Kommunikation". 29

Wenn McDowell davon ausgeht, dass Wahrnehmen immer ein Fallsein der Welt meint, auf das gezeigt werden kann wie auf Dinge, so setzt er damit einen Begriff von Wahrnehmen voraus, der den Akt des Wahrnehmens und die wahrnehmbare Welt eingrenzt. Nur das Gegebene in der Form der Gegenständlichkeit gilt als das in der Anschauung Gegebene. Für den Begriff der Welt heißt das, dass immer nur Dinge erfasst werden, auf die gezeigt werden kann, indem geurteilt werden kann, ,that things are thus and so“. Dazu kommt eine Universalität dieser Weltsicht als Allartikulierbarkeit: „No aspect is unnameable $(\ldots)$ “. 30

Die sinnlich wahrnehmbare Welt des Leiblichen jedoch, die Schmitz hervorhebt, geht nicht in Zeigbarkeit auf. Weil letztlich allein die „unwillkürliche Lebenserfahrung“ für Schmitz' Behauptungen rechtfertigt, sind diese immer als doppelt relativ anzusehen. Urteile sind abhängig von der Situation von jemand Bestimmten zu einem bestimmten Moment. ${ }^{31}$ Situationen wiederum sind von einer kulturellen „Abstraktionsbasis“ mitkonditioniert, die etwas als Fall von etwas zu verstehen erlaubt. Eine genaue Zeigbarkeit von Fakten gibt es daher nicht, weil diese erst in einer Hinsicht an Eindeutigkeit gewinnen. Da das Erfassen der Sachen als einzelne Sachen immer aus einer Perspektive erfolgt, benutzt Schmitz den Begriff ,Sachverhalte“. 32

Tatsächlichkeit als propositional strukturiertes Wissen in der Form „dass x“, kommt in dieser Klarheit zwar auch bei ihm vor. Dabei differenziert Schmitz

23

MW, S. 56f.

24

Wie so oft orientiert sich McDowell hierbei an Evans neofregeanischem Denken. Vgl. G. Evans, The Varieties of Reference, S. 64, 71.

25

John Henry McDowell, ,Truth-Value Gaps“, Meaning, Knowledge, and Reality, Harvard University Press, Cambridge (MA) 1998, S. 199-213, hier S. 204. McDowell orientiert sich bei seiner Idee der Gegenstandsabhängigkeit des Begriffs an Russell und Frege.

26

MW, S. 19.

27

Ebd., S. 39.

28

Ebd., S. 103.

29

Das ,eigenleibliche Spüren“ ist für Schmitz

Teil des übergeordneten Phänomens der „leiblichen Kommunikation“, bei der Leib und Welt „Koagieren ohne Reaktionszeit" (Hermann Schmitz, Der unerschöpfliche Gegenstand. Grundzüge der Philosophie, Bouvier, Bonn 1990, S. 137). Diese ,leibliche Kommunikation" ersetzt für Schmitz das Verständnis eines intentionalen Bezugs zur
Welt. Vgl. Hermann Schmitz, Subjektivität, Bouvier, Bonn 1968, S. 3, 12 f.

30

Dieser Punkt der jederzeitigenArtikulierbarkeit gilt auch noch in der aktuellen Formulierung seines Modells, bei der McDowell seine These des propositionalen Gehalt von Erfahrungen zu einem nicht-diskursiven, konzeptionellen abschwächt: ,But the conceptual content that allows us to avoid the Myth is intuitional, not propositional, so experiencing is not taking things to be so. In bringing our surroundings into view, experiences entitle us to take things to be so; whether we do is a further question." Siehe: John Henry McDowell, ,Avoiding the Myth of the Given", Having the World in View. Essays on Kant, Hegel and Sellars, Harvard University Press, Cambridge (MA) 2009, S. 256-272; hier: S. 269. John Henry McDowell, „What Myth?", The Engaged Intellect, Harvard University Press, Cambridge (MA) 2009, S. 308-323; hier: S. 320.

31

Vgl. Hermann Schmitz, Kurze Einführung in die Neue Phänomenologie, Karl Alber Verlag, Freiburg 2009, S. 13ff.

32

Vgl. beispielsweise: H. Schmitz, Höhlengänge. Über die gegenwärtige Aufgabe der Philosophie, S. 36. 
aber zwischen „subjektiven“ und „objektiven Tatsachen“. ${ }^{33}$ Letztere besitzen die Allgemeinheit und Artikulierbarkeit, die McDowells positivistischen Begriff von Faktizität bestimmt. „Subjektive Tatsachen“ gelten jedoch nur für ein Subjekt und verlieren ihre Bedeutung, wenn sie als Satzaussagen - Wie das berüchtigte Beispiel von Hermann Schmitz: „Hermann Schmitz ist traurig.“ - zu einem zeigbaren, für jedermann jederzeit zugänglichen „Dies da!“ vergegenständlicht werden. Derartige Sätze können nur von außen wie eine Decke über ein ,affektives Betroffensein“ geworfen werden. Die Vielsagbarkeit der Realität als „subjektive Tatsache“ ist somit keine idiosynkratische Vernebelung von Tatsachen, die klar gestellt werden können, sondern die zwiespältige Natur der Dinge selbst. ${ }^{34}$

Damit steht der Neuen Phänomenologie aber auch kein intersubjektiver Geltungsanspruch und auch keine experimentelle Nachprüfbarkeit für ihre Urteile zur Verfügung. Anstelle von festen Überzeugungen, die andere Überzeugungen beeinflussen können, besitzen die Tatsachenaussagen der Neuen Phänomenologie eine Unsicherheit. Es kann die Evidenz der „,subjektiven Tatsachen" nicht in einem Raum der Gründe als für jedermann jederzeit zugängliche Tatsache einen überzeugenden Grund manifestieren. So ist es etwa nicht möglich, atmosphärische Gefühle als Urteil zu artikulieren und sie als „bits of the Given“" 35 mit der Geltung von Urteilen in Begründungen zu verwenden. Betroffensein verpflichtet nur für jemand Bestimmten zu einem bestimmten Moment.

Gegenüber dieser konstitutiven Unsicherheit im Verständnis des Erkennens der Neuen Phänomenologie erscheint McDowells Ansatz zu grob. McDowells Begriff des zeigenden Wahrnehmens lässt sich neuphänomenologisch allenfalls auf „,ungefühlte Gefühle“ 36 beziehen. Der weite und basale Bereich der „subjektiven Tatsachen“ kann in McDowells Konzept nicht auftauchen. Sein Wahrnehmen kennt kein Nahegehen. Sein Versuch, das Wahrnehmen als subjektives Erleben rational aufzuwerten, verläuft daher innerhalb des Rahmens einer leiblich neutralisierten Subjektivität. Das Bild eines reibungslosen Rotierens in einer Leere, das McDowell für ein vom Wahrnehmen verlassenes Denken gebraucht, trifft zum großen Teil auf seine Philosophie selbst zu.

Aus diesem Mangel in McDowells Konzeption kann von der Neuen Phänomenologie her auch eine tiefere Kritik erfolgen. Obwohl sich McDowell dafür ausspricht, den Objektivismus der Naturwissenschaften zu relativieren, indem er ihn als eine unbemerkte Übernahme der skeptizistischen Prämissen verständlich macht, ähnelt sein Bild der Wahrnehmung dem des Szientismus. In dem Entwurf seines ,minimalen Empirismus“ gleicht die Orientierung an zeigbaren, für jeden fasslichen Stücke einer für jeden geltenden gegebenen Umwelt, den Standardisierten, neutralisierenden Beobachtungen der neuzeitlichen Wissenschaft. Diese nach dem Primat der statistischen Messbarkeit von einzelnen, selektierbaren Merkmalen ausgehende Wahrnehmungsbegriff, hat Schmitz als Teil der ,psychologistisch-reduktionistisch-introjektionistischen Vergegenständlichung“" kritisiert, die er als „Abstraktionsbasis“ des Abendlandes, ausgehend von Demokrit, begreift. ${ }^{37}$

So fehlt an McDowells Modell nicht nur die Dimension „Nahegehen“. Als blindes Befolgen des naturalistischen Paradigmas unterdrückt es auch den Bereich der wahrnehmbaren Welt, der einem nahegehen kann. McDowells therapeutische Sichtweise einer ,fallible openness" ${ }^{\text {"38 }}$ stellt somit, weil sie einseitig auf die Form „Zeigen“ ausgelegt ist, keine „unproblematic openness to the world“39 dar. Im Ganzen führt McDowells „minimaler Empirismus“ 
damit zu einem minimalistischen Begriff von Empirie, der die sinnlich wahrnehmbare Welt empfindlich reduziert. Für diese gilt: Having the world only as a view in view. ${ }^{40}$

Von hier aus lässt sich dann der Unterschied im Verhältnis von Geist und Welt fassen. Während McDowell das Wahrnehmen als eine Reibung zur Welt versteht, geht es Schmitz mehr um eine Welt, die als Resonanz und Ergriffenheit erfahren wird. Bei McDowell spielt die wahrgenommene Welt die Aufgabe eines Richters in einem Tribunal, bei dem Urteile von der Wahrnehmung gerechtfertigt werden, weil schon Wahrnehmungen urteilsförmig zu verstehen sind. Auch bei Schmitz besitzen Wahrnehmungen einen normativen Wert. Dieser äußert sich aber in einer pathischen Bindung des Geistes, deren Kontur viel unsicherer zu explizieren ist. Die Welt, die einen angeht, ist bei Schmitz nicht einfach positiv vorhanden, sondern sie entsteht erst in einem Sichfinden.

Dazu gehört ein leibliches Aussetzen in einer Umgebung und damit eine passive Rolle des Geistes. Diese Dimension des Findens wird insbesondere in Bezug auf Gefühle deutlich. Derartige ,subjektive Tatsachen“ gehen einen an und besitzen für Schmitz die Autorität der Überwältigung:

„Wie mir scheint, besteht für jedes Gefühl eine notwendige Bedingung seiner so verstandenen Echtheit bei jedem Subjekt darin, daß das Subjekt ihm wenigstens zeitweise nicht gewachsen ist, sondern von ihm überwältigt und gezwungen wird, sich ihm preiszugeben, wenn es sich auch anschließend diesem von dem Gefühl ausgeübten Zwang wieder entzieht. “" ${ }^{11}$

33

Vgl. Hermann Schmitz, System der Philosophie, Bd. 3, Teil 2, Der Gefühlsraum, Bouvier, Bonn 1969, S. 50f.

34

Schmitz setzt sich gerade in Bezug auf Heideggers Ansatz bei einem ,sich zeigen“ kritisch von der Tradition der Phänomenologie ab. Vgl. H. Schmitz, Kurze Einführung in die Neue Phänomenologie, S. 13.

35

MW, S. 19.

36

Bei einem Aufeinandertreffen von Gefühlen kann es laut Schmitz auch dazu kommen, dass das Erfahren des Gefühls nicht die Intensität des Betroffenseins erreicht. Er spricht von einem „Fühlen des Gefühls“. Vgl. etwa Hermann Schmitz, Was ist Neue Phänomenologie?, Ingo Koch, Rostock 2003, S. 44. Es handelt sich dabei um Gefühle, die in ,eigenleiblicher Resonanz" auf Gefühle reagieren, die in der Umgebung des Subjekts auftreten. In ihnen kommt es nicht zu einem sinnlichen Erleben als ,affektives Betroffensein“, das mit der Macht des Ergreifens wirkt. Schmitz bezeichnet derartige Gefühle auch als ,ungefühlte Gefühle“, die nur eine ,sekundäre Anteilnahme“ entfalten. Vgl. H. Schmitz, Der Gefühlsraum, S. 137, 154.

37

Demgegenüber spricht sich Schmitz für eine typologische Erfassung aus, die im Geiste
Goethes nach markanten Charakteristika fahndet, ohne eine spürende Subjektivität bei diesen Abstraktionen außen vor lassen zu müssen. 38

MW, S. 143.

39

Ebd., S. 155.

40

McDowell steht damit in der philosophischen Tradition, die als vorherrschendes Paradigma für ein Verständnis des sinnlichen Erkennens das Sehen als Leitsinn nimmt. Es finden sich bei McDowell zahlreiche Stellen, die dafür als Beleg gelten können. Etwa: „An Anschauung is a having in view." Siehe: J. H. McDowell, „Avoiding the Myth of the Given“, S. 260. McDowell erwähnt selbst, dass in der Philosophie das Paradigma des Sehens zumeist selbstverständlich die Thematisierung des Verständnisses von sinnlicher Wahrnehmung prägt. Vgl. ebd. Er stößt sich daran nicht, doch genau daran stößt sich die Neue Phänomenologie.

41

H. Schmitz, Der Gefühlsraum, S. 141. Vgl. auch die folgende Bemerkung: „Es gibt keine Gefühle, die ich habe, sondern nur Gefühle, die mich haben; sie sind nicht subjektive Akte, sondern übersubjektive Mächte, die die Weite, in der wir leben, gleichsam atmosphärisch durchziehen, über uns kommen und uns mit sich reißen wie die Winde." Siehe: Hermann Schmitz, Subjektivität, Bouvier, Bonn 1968, S. 23. 
Geist und Welt durchdringen sich nach Schmitz leiblich miteinander. Die Welt ist somit alles, was den Geist als „leibliche Kommunikation“" auf vielfache Weise in den Bann ziehen kann. Sie ist dämonisches Gebiet, um eine Bemerkung Thomas Manns in Bezug auf die Musik auf eine leiblich verstandene Welt zu übertragen. Diese dämonische Welt gilt es erst einmal in ihrer Fülle gelten zu lassen und an ihr teilzunehmen. ${ }^{42}$ Der Geist muss sich von der Welt überwältigen lassen, um sie zu verstehen.

Doch auch bei den von der Gefühlsüberwältigung distanzierten Urteilen ist sich der Phänomenologe, so wie ihn Schmitz versteht, bewusst, dass eine satzförmige Explikation von „subjektiven Tatsachen“, anders als es McDowell sieht, niemals vollständig sein kann. Den Reichtum eines affektiven Betroffenseins können Aussagen nicht wiedergeben, da das sinnliche Erleben nicht bloß in der Struktur von Feinkörnigkeit zu erfassen ist. Die Welt ist keine Ansammlung von Wahrnehmungskörnern, von ,particular bits of the Given", ${ }^{43}$ die der Geist wie ein Huhn vollständig als Urteile aufpicken kann. Der Geist, so wie ihn Schmitz sieht, gleicht eher einem Argonauten, der sich auf unsicherer Fahrt befindet und sich dabei bemüht, die Strömungen und Winde, die ihn zwiespältig bestimmen und ergreifen, mitzunotieren und verständlich zu typisieren.

Mit dieser Kritik an McDowell von der Position der Neuen Phänomenologie her könnte ein Vergleich der beiden Philosophien enden. McDowells Denken zeigt sich als ein ungenügender Versuch, die Prämissen der analytischen Philosophie so zu erweitern, dass sie offen werden für das sinnliche Erleben. Auch seine therapeutische Absicht, Welt und Geist als verbunden dazustellen, macht es sich aus der Sicht der Neuen Phänomenologie zu leicht, da sie den Großteil der wahrnehmbaren Welt nicht umfasst. Zwei Gedanken von McDowells Philosophie könnten allerdings dennoch fruchtbar werden für eine zukünftige Auseinandersetzung von neuphänomenologischen und analytischen Positionen.

\subsection{Aua!}

Ein möglicher Hinweis auf einen produktiven Dialog zwischen den Philosophien von McDowell und Schmitz könnte McDowells Betonung der Situationsgebundenheit des Gebrauchs der Begriffe sein. Die Idee des ,,singulären Gedankens" könnte dazu führen, den Begriff der neuphänomenologischen Explikation zu verdeutlichen. Dadurch wäre es möglich, einen weiter gefassten Begriff von Sachlichkeit und Präzision zu erlangen, der sich auch der Tradition des dichtenden Denkens nicht mehr so resolut verschließt, wie es Schmitz tut.

Wie die Begriffe bei McDowell als ein wahrnehmendes Zeigen zu begreifen sind, sind sie in einem weiteren Verständnis von sinnlicher Wahrnehmung - zum Beispiel in Bezug auf Atmosphären - als ein wahrnehmendes Spüren zu deuten. Der singuläre Gehalt einer Atmosphäre kann so verstanden werden, dass er sich in dem Bilden von Begriffen unter dem Einfluss des sinnlichen Erlebens einer bestimmten affektiven Betroffensein äußert. Die Welt käme als Betroffensein von Atmosphären zur Sprache und nicht aus dem Off einer distanzierten Nachträglichkeit. Im Beschreiben wäre so das Betroffensein eines Sichfindens anwesend. Daher wird durch diesen atmosphärischen Begriff des ,singular thought“ nicht einfach nur die Anschaulichkeit im Argumentieren erhöht, sondern eine Unmittelbarkeit zur Welt erst herbeigeführt und demonstriert. 
Das Besondere eines derartigen Verständnisses von begrifflicher Artikulation ist ihre rhetorische Qualität. Ein kommentierendes Hinweisen auf Atmosphären benötigt durch die affektive sinnliche Präsenz die Legitimation zu einem umfassenderen Ausdrücken, das eine bloße Feinkörnigkeit übertrifft. Dieses expressive Explizieren erreichte seine Präzision in einem Vermitteln des qualitativen Erlebens einer Situation. ${ }^{44}$

Eine schöne Formulierung über die Wirkung eines derartigen singulären Explizierens findet sich in Goethes Roman Die Wahlverwandtschaften. Dort heißt es über einen Kenner von Parkanlagen:

„Sein geübtes Auge empfing jeden Effekt ganz frisch (...). Man kann wohl sagen, daß durch seine Bemerkungen der Park wuchs und sich bereicherte. “45

Der oftmals sehr wissenschaftliche und auf eine Kritik an der Tradition ausgerichtete Gestus der Neuen Phänomenologie könnte durch einen von McDowells Idee der „singulären Gedanken“ abgeleiteten evokativen Gebrauch von Begriffen durchlässiger werden. Damit wäre einer Tendenz zu folgen, die Schmitz in seinen Schriften darstellte. Seine Gedanken sind immer reichhaltig durch Beispiele aus dem Alltag und der Literatur illustriert. Es wäre vorstellbar, dass sich diese Anschaulichkeit durch eine Anerkennung auch der hybriden Tradition der essayistischen und literarischen Formen der Philosophie erweitert. Möglich wäre so auch ein stärkeres Hineinspüren in die „Gefühlsräume" und Ausdrucksformen des Zeitgeistes. Schmitz hat sich allerdings negativ gegenüber einem evokativen Gebrauch der Begriffe geäußert:

„Wenn ich einen Gegenstandstyp aus dem Gegenstandsbereich des eigenleiblichen Spürens charakterisiere, z. B. den Schmerz als einen Konflikt im vitalen Antrieb, (...) denke ich gar nicht daran, den Leser in die Naivität eines vorintentionalen Schmerzerlebens zu versetzen, das unmittelbar, aller Reflexion ledig, nur noch den Schmerz auszusprechen hätte; dafür wäre es besser, auf Phänomenologie zu verzichten und schlicht ,Aua!' zu rufen. “46

42

Die Nichtpartizipation an der Welt ist auch an dem Verständnis von Gefühle ablesbar, wie er in der Philosophie des Geistes geführt wird. Dort kam es etwa seit den 60er Jahren im englischsprachigen Raum und um das Jahr 2000 in Deutschland zu einer Neubewertung und Neubeachtung von Emotionalität. Unterschieden wird in diesem Diskurs zwischen „emotions“ und ,feelings“. Während ,feelings" allein eine Erlebnisqualität besitzen, gelten ,emotions“ als anspruchsvollere kognitive Zustände, die sich auf etwas richten und es in einer spezifischen Weise als seiend repräsentieren: „Emotions besitzen so in ihrem phänomenalen Erleben einen geistigen Gehalt." Vgl. Sabine A. Döring, Philosophie der Gefühle, Suhrkamp, Frankfurt a. M. 2009 , S. 12ff. Erst diese nonverbale normative Haltung gibt den Gefühlen einen epistemischen Wert. So heißt es bei Döring, die sich auf den Sprachgebrauch der englischen Diskurse bezieht: „Gefühle im engeren Sinne sind also Emotionen bzw. ,emotionale Gefühle"."(Ebd., S. 14). Schmitz unterläuft die Unterscheidung von ,emotions" und ,feelings“, indem er sie in einen größeren Zusammenhang integriert. Zwar kennt auch Schmitz eine Intentionalität von Gefühlen. Bei ihm stellen sie aber lediglich eine Schicht von Emotionalität dar, die er als „Erregungen“ bezeichnet. Für eine allmähliche Wahrnehmung der Neuen Phänomenologie im emotionsphilosophischen Diskurs spricht etwa der Sammelband Gefühle als Atmosphären. Neue Phänomenologie und philosophische Emotionstheorie, der 2011 in Berlin von Andermann und Eberlein herausgegeben wurde.

43

MW, S. 19.

44

Vgl. dazu eine tiefsinnige Formulierung von Gadamer: ,Ein jedes Wort läßt daher auch, als das Geschehen eines Augenblicks, das Ungesagte mit da sein, auf das es sich antwortend und winkend bezieht." Hans-Georg Gadamer, Wahrheit und Methode. Grundzüge einer philosophischen Hermeneutik, Mohr, Tübingen 1990, S. 462.

45

Johann Wolfgang von Goethe, Die Wahlverwandschaften, Gutenberg Verlag, Hamburg 1965, S. 429f.

46

H. Schmitz, Was ist Neue Phänomenologie?, S. 408 . 


\subsection{Make it implicit!}

Ein möglicher zweiter Punkt für eine produktive Verbindung von McDowell und Schmitz könnte in der Betonung der Passivität der Wahrnehmung liegen. Dieser von McDowell erwähnte, aber erst durch die Konzepte der Neuen Phänomenologie in seiner Bedeutung verständliche Gedanke, bekäme einen Sinn als methodische Betonung des partizipativen Momentes des Geistes am Leib.

Die Gefahr einer Verstellung der leiblichen Wirklichkeit liegt nicht erst auf der Ebene der Artikulation, sondern schon auf der Ebene der Wahrnehmung als eines zeigenden Bestimmens. Dies ergibt sich durch die kritische Analyse von McDowells Verständnis der Wahrnehmung als eines Zeigens. Es wird deutlich, dass ein Zeigen reduktive Wirkungen besitzen kann. McDowells Idee der Kooperation des sinnlichen Erlebens mit dem urteilenden Denken betont indirekt allerdings die Notwendigkeit einer Explikation hin zu dem Punkt eines zeigbaren Gehaltes, auch wenn er sie auf der ausdrücklichen Ebene seiner Gedanken übertreibt. Erst diese Zuspitzung einer explikativen Zeigbarkeit erlaubt eine präzise, dauernde und kommunikative Verständigung über die Tatsächlichkeit.

Schmitz' Definition der Aufgabe der Philosophie als ein „Sichbesinnen auf sein Sichfinden in seiner Umgebung“" könnte so noch einmal präzisiert werden. Um den Unterschied zu Formen der Explikation in der analytischen Philosophie herauszustellen und doch die Bestimmtheit einer zeigenden Ausdrücklichkeit nicht zu verlieren, könnte der Begriff des Sichbesinnens um die Bedeutungsnuance eines ausdrücklich unternommenen „Sichbesinnens“ ergänzt werden. Ein ,eigenleibliches Spüren“ wäre demnach ein explizites Implizitsein, das der Autorität von Gefühlen nachdrücklich offensteht und folgt. Ein Verwenden von Begriffen und ein Wechsel in die Wahrnehmungshaltung eines Zeigens wäre dabei bewusst auszusetzen.

Ein in der analytischen Philosophie berühmtes Buch von Robert Brandom trägt den Titel: Make it explicit!. Der Tendenz nach verfolgt auch die Neue Phänomenologie dieses Programm. Um ihre Erdung in leiblichen Erfahrungen als Passivität des Geistes aber zu betonen, verliefe ihre analytische Ausrichtung in einem ersten Schritt aber eine andere, die dem Slogan folgte: „Make it implicit!“

\section{Der Welt treu bleiben}

Durch das Denken von Hermann Schmitz lernt die Vernunft wieder spürend einen Halt in ihren dem bloßen Wollen unverfügbaren Lebenserfahrungen zu finden. Gerade diese Passivität kann als Reflexion der Bewegungsabläufe in der Moderne gesehen werden. An die Stelle des bloßen Vorwärts eines blinden Aktivismus, den engen Kreisläufen überkritischer Theorien oder scholastischer Analysespiele, tritt eine Leibbesinnlichkeit. Diese ist zu verstehen als eine somatische Kontemplation, die immer wieder organisch in ein Denken ausschwingt. Die Passivität der neuphänomenologischen Haltung ist als ein waches, zugleich unschuldiges und redliches Mitgehen in den unwillkürlichen Engungen und Weitungen zwischen Leib, Geist und Welt zu begreifen. Damit wird der moderne Selbstzweifel des Geistes ins Tätige gewendet. Für das neuphänomenologische Denken gilt: Die Wirklichkeit wurde genug interpretiert, verändert oder als Abstand beklagt. Es kommt darauf an, sich ihr hinzugeben und ihre Fülle zu entdecken. Das Wahrnehmen ist hierbei die Frömmigkeit des Denkens. 
Die Neue Phänomenologie befreit so von der Enge einer Rationalkultur, wie sie paradigmatisch in der analytischen Philosophie auftritt. Wie eine verlorene Raumstation, die auf einmal auf den Schirmen der Überwachungsgeräte erscheint, nähert sich das Denken in der Neuen Phänomenologie wieder einer Umlaufbahn um die Erde. Von dort oben funkt der Astronaut Schmitz den Erdbewohnern, die der Erde fremder geworden sind als es Raumfahrer je waren, Beschreibungen ihrer Umgebung zu. Ein neuphänomenologisches Beschreiben weist den außerirdischen Innerirdischen auf ein mögliches Sichfinden in volleren, aber verdeckteren Wirklichkeiten hin. Es taucht mit der frohen Botschaft auf, dass es doch eine Wirklichkeit gibt, die uns angeht und die es - wieder einmal - neu zu entdecken gilt. Die Unergriffenheit ist nicht alles. Es gibt mehr als ein Davor. Das süchtige Starren auf Geräte, die Welt liefern sollen und das verdächtigende Belauern fremder Außenwelten, brauchen nicht die letzte Antwort des Geistes auf den Abstand zur Welt zu sein. Zu der gegenwärtigen Aufgabe der Philosophie schreibt Schmitz daher:

,[Es ist nötig] für die funktionslos und autoaggressiv gewordene Hybris der klassischen Philosophie ein Gegengift zu finden, das die Hindernisse für ein unverkünsteltes Sichbesinnen des Menschen auf ein Sichfinden in seiner nicht mehr reduktionistisch entleerten Umgebung auflöst. (...) Die Philosophie kann damit aber nicht schon eine Regeneration der verschütteten Möglichkeiten der Lebensgestaltung erwirken. Ihr bleibt nur die Aussicht, durch genaue und schmiegsame Begriffsbildung eine Sprache vorzubereiten, die die Menschen näher an das heranführt, was sie leben können und wie ihnen zumute ist. “47

$\mathrm{Zu}$ dieser Selbstbesinnung der Philosophie auf ihr Sichfinden gehört auch ein philosophischer Kontinentaldrift. Die angelsächsische Philosophie hätte sich wieder stärker zu kontinentalisieren. Das Werk McDowells ist dafür ein Beispiel. Vor allem aber hätte sich die kontinentale Philosophie wieder selbstbewusster zu europäisieren und von der analytischen Philosophie zu distanzieren. Dafür ist das Denken von Hermann Schmitz ein Vorbild.

Die Neue Phänomenologie wird später einmal, so kann gemutmasst werden, Teil der somatischen Renaissance des Geistes sein, die mit Kierkegaards Kritik an Hegel langsam einsetzte, mit Nietzsche und Heidegger erste radikale Höhen erreichte und in den Werken von Sloterdijk und Schmitz in ein begehbares Hochplateau übergeht. Sie ist Teil der Tradition, die aus der Tradition des Substanzdenkens heraustritt. Das ehemals Akzidentelle rückt bei ihr ins Zentrum und eröffnet eine Sicht auf Objektivität, die ephemer, momentan, diffus, fragil, zufällig und vor allem energetisch sein darf. Das Werk Hermann Schmitz' stellt damit in Zukunft einen der Riesen dar, den die Zwerge des Denkens zu erklettern haben, um auf seinen Schultern überhaupt den unverstellten Blick richten zu können auf die Sachen, die uns angehen und ansprechen.

Eine Philosophie der Zukunft wird immer auch neuphänomenologisch sein, sie wird aber mehr sein müssen als Neue Phänomenologie. Der Grund dafür liegt in dem Leibzentrismus der Neuen Phänomenologie. Zwar rehabilitiert Schmitzs Philosophie den passivistischen Anteil der vormodernen Denkkultur. Dabei kommt es aber zu einer Somatisierung der Kontemplation. Mit ihrer Orientierung an dem durchschnittlichen Erfahrungshorizont zeigt die Neue Phänomenologie die Tendenz, die thanatophile Kontemplation, die nicht nur im Abendland das Kriterium für eine Suche nach der Wahrheit als höheres Leben bildet, allein als Regime einer leiblosen Vernunft gelten zu lassen. Diese

47

H. Schmitz, Höhlengänge. Über die gegen-

wärtige Aufgabe der Philosophie, S. 33. 
hochkulturellen Spannungen waren aber immer auch Versuche, von dem Sittenregime des Alltagsbewusstseins, den giftigen Atmosphären von Epochen, den Entseelungen des Innenlebens loszukommen. Ein metaphysisches Sichbesinnen strebt danach, die Automatismen des Denkens zu brechen und sich zu immunisieren gegen eine fremde Außenwelt, um offener zu werden für die Realität. ${ }^{48}$ Die Frage wird sein, wie die Neue Phänomenologie diese leibformende Passivität in ihr Denken wird integrieren können, die etwa Rilke im Stunden-Buch anklingen lässt:

„Wenn es nur einmal so ganz stille wäre.

Wenn das Zufällige und Ungefähre

verstummte und das nachbarliche Lachen,

wenn das Geräusch, das meine Sinne machen,

mich nicht mehr so sehr verhinderte am Wachen -:

Dann könnte ich in einem tausendfachen

Gedanken bis an deinen Rand dich denken und dich besitzen (nur ein Lächeln lang), um dich an alles Leben zu verschenken wie einen Dank." ${ }^{49}$

Michael Meyer

\section{Pronalaženje neizgubljenog svijeta}

Napomene o komparaciji filozofijā

Hermanna Schmitza i Johna McDowella

\section{Sažetak}

Filozofiju Johna McDowella i novofenomenološko mišljenje Hermanna Schmitza možemo shvatiti kao pokušaje terapije distance između duha [uma] i svijeta koja u modernom vremenu poprima dramatične razmjere. U ovom članku autor pokušava usporediti terapeutske koncepte Schmitza i McDowella, uzimajući u obzir moderno doba i posebno kontrast između fenomenološkog $i$ analitičkog načina filozofiranja. Glavna razlika u njihovim pokušajima da predstave otvorenost duha [uma] prema svijetu jest ta što Schmitz između duha [uma] i svijeta umeće (živo) tijelo (Leib) kao centralnu veličinu. Naspram takvog bogatijeg pojma opažanja u većoj mjeri u oči upada redukcionizam McDowellova modela. Ipak, autor u ovom članku ukazuje na dvije mogućnosti da se i McDowellove ideje učine plodnim i u novofenomenološkom kontekstu.

\section{Ključne riječi}

Hermann Schmitz, John McDowell, nova fenomenologija, otvorenost prema svijetu, skepticizam, moderna 


\title{
Michael Meyer
}

\section{Finding the World That Was Not Lost \\ Remarks on Comparing the Philosophies

of Hermann Schmitz and John McDowell

\begin{abstract}
John McDowell's philosophy and Hermann Schmitz's new phenomenological thinking can be considered to be a therapeutic attempt on the distance between spirit [mind] and world that is taking dramatic proportions in modern time. In this paper, the author is trying to compare Schmitz's and McDowell's therapeutical concepts while taking into consideration the modern age and especially the contrast between the phenomenological and analytical way of philosophising. The main difference in their attempts to present the openness of the spirit [mind] towards the world is that Schmitz sets the living body (Leib) of central importance between spirit [mind] and world. In difference to Schmitz's rich notion of perception, one can easily notice McDowell's reductionism. Nonetheless, in this paper, the author points at the two possibilities by which McDowell's ideas might seem fruitful in new phenomenological context.
\end{abstract}

\section{Keywords}

Hermann Schmitz, John McDowell, New phenomenology, openness towards world, scepticism, modernity

\section{Michael Meyer \\ La découverte d'un monde non perdu \\ Remarques sur la comparaison des philosophies de Hermann Schmitz et John McDowell}

\begin{abstract}
Résumé
La philosophie de John McDowell et la pensée de la Nouvelle Phénoménologie de Hermann Schmitz se laissent comprendre comme des tentatives de thérapie de mise à distance entre l'esprit et le monde, qui, au sein du monde moderne, ont pris une ampleur dramatique. Dans cette article, l'auteur tente de comparer les concepts thérapeutiques de Schmitz et de McDowell en prenant en considération les temps modernes, et plus particulièrement le contraste existant entre l'approche analytique et phénoménologique de philosopher. La différence principale au sein de leurs tentatives visant à présenter l'ouverture de l'esprit envers le monde résulte du fait que Schmitz introduit le corps (Leib) vivant entre l'esprit et le monde en tant que grandeur centrale. Face à ce concept de la perception, le réductionnisme du modèle de McDowell n'en est que plus frappant. Néanmoins, l'auteur de cette article met en avant deux possibilités, rendre les idées de McDowell fécondes, et cela dans le contexte de la Nouvelle Phénoménologie.
\end{abstract}

\section{Mots-clés}

Hermann Schmitz, John McDowell, Nouvelle Phénoménologie, ouverture envers le monde, scepticisme, époque moderne

Sloterdijks Ansätze zu einer immunologischen Ontologie könnten als ein Versuch angesehen werden, den Begriff Weltoffenheit avancierter zu denken: ,Metaphysik ist eine Antwort auf Weltoffenheit - Weltoffenheit kann nur durch sphärische Sicherungen lebbar gemacht werden. Sicherungen sind ihrerseits immer von neuem am Offenen zu orientieren." Siehe: P. Sloterdijk, Was geschah im 20. Jahrhundert?, S. 172 .
49

Rainer Maria Rilke, Das Stunden-Buch Insel, Frankfurt a. M. 2000, S. 15. 